\title{
A note on poly-Bernoulli numbers and polynomials of the second kind
}

Taekyun Kim ${ }^{1 *}$, Hyuck In Kwon ${ }^{1}$, Sang Hun Lee ${ }^{2}$ and Jong Jin Seo ${ }^{3}$

"Correspondence: tkkim@kw.ac.kr 'Department of Mathematics, Kwangwoon University, Seoul, 139-701, Republic of Korea Full list of author information is available at the end of the article

\section{Abstract \\ In this paper, we consider the poly-Bernoulli numbers and polynomials of the second kind and presents new and explicit formulas for calculating the poly-Bernoulli numbers of the second kind and the Stirling numbers of the second kind.}

Keywords: Bernoulli polynomials of the second kind; poly-Bernoulli numbers and polynomials; Stirling number of the second kind

\section{Introduction}

As is well known, the Bernoulli polynomials of the second kind are defined by the generating function to be

$$
\frac{t}{\log (1+t)}(1+t)^{x}=\sum_{n=0}^{\infty} b_{n}(x) \frac{t^{n}}{n !} \quad(\text { see }[1-3])
$$

When $x=0, b_{n}=b_{n}(0)$ are called the Bernoulli numbers of the second kind. The first few Bernoulli numbers $b_{n}$ of the second kind are $b_{0}=1, b_{1}=1 / 2, b_{2}=-1 / 12, b_{3}=1 / 24$, $b_{4}=-19 / 720, b_{5}=3 / 160, \ldots$.

From (1), we have

$$
b_{n}(x)=\sum_{l=0}^{n}\left(\begin{array}{l}
n \\
l
\end{array}\right) b_{l}(x)_{n-l},
$$

where $(x)_{n}=x(x-1) \cdots(x-n+1)(n \geqq 0)$. The Stirling number of the second kind is defined by

$$
x^{n}=\sum_{l=0}^{n} S_{2}(n, l)(x)_{l} \quad(n \geqq 0) .
$$

The ordinary Bernoulli polynomials are given by

$$
\frac{t}{e^{t}-1} e^{x t}=\sum_{n=0}^{\infty} B_{n}(x) \frac{t^{n}}{n !} \quad(\text { see }[1-18])
$$

When $x=0, B_{n}=B_{n}(0)$ are called Bernoulli numbers.

\section{Springer}

@2014 Kim et al.; licensee Springer. This is an Open Access article distributed under the terms of the Creative Commons Attribution License (http://creativecommons.org/licenses/by/2.0), which permits unrestricted use, distribution, and reproduction in any medium, provided the original work is properly cited. 
It is well known that the classical poly-logarithmic function $\operatorname{Li}_{k}(x)$ is given by

$$
\operatorname{Li}_{k}(x)=\sum_{n=1}^{\infty} \frac{x^{n}}{n^{k}} \quad(k \in \mathbb{Z})(\text { see }[8-10])
$$

For $k=1, \mathrm{Li}_{1}(x)=\sum_{n=1}^{\infty} \frac{x^{n}}{n}=-\log (1-x)$. The Stirling number of the first kind is defined by

$$
(x)_{n}=\sum_{l=0}^{n} S_{1}(n, l) x^{l} \quad(n \geq 0)(\text { see }[16]) .
$$

In this paper, we consider the poly-Bernoulli numbers and polynomials of the second kind and presents new and explicit formulas for calculating the poly-Bernoulli number and polynomial and the Stirling number of the second kind.

\section{Poly-Bernoulli numbers and polynomials of the second kind}

For $k \in \mathbb{Z}$, we consider the poly-Bernoulli polynomials $b_{n}^{(k)}(x)$ of the second kind:

$$
\frac{\operatorname{Li}_{k}\left(1-e^{-t}\right)}{\log (1+t)}(1+t)^{x}=\sum_{n=0}^{\infty} b_{n}^{(k)}(x) \frac{t^{n}}{n !}
$$

When $x=0, b_{n}^{(k)}=b_{n}^{(k)}(0)$ are called the poly-Bernoulli numbers of the second kind.

Indeed, for $k=1$, we have

$$
\frac{\operatorname{Li}_{k}\left(1-e^{-t}\right)}{\log (1+t)}(1+t)^{x}=\frac{t}{\log (1+t)}(1+t)^{x}=\sum_{n=0}^{\infty} b_{n}(x) \frac{t^{n}}{n !}
$$

By (7) and (8), we get

$$
b_{n}^{(1)}(x)=b_{n}(x) \quad(n \geq 0) .
$$

It is well known that

$$
\frac{t(1+t)^{x-1}}{\log (1+t)}=\sum_{n=0}^{\infty} B_{n}^{(n)}(x) \frac{t^{n}}{n !}
$$

where $B_{n}^{(\alpha)}(x)$ are the Bernoulli polynomials of order $\alpha$ which are given by the generating function to be

$$
\left(\frac{t}{e^{t}-1}\right)^{\alpha} e^{x t}=\sum_{n=0}^{\infty} B_{n}^{(\alpha)}(x) \frac{t^{n}}{n !} \quad(\text { see }[1-18]) .
$$

By (1) and (10), we get

$$
b_{n}(x)=B_{n}^{(n)}(x+1) \quad(n \geq 0) .
$$


Now, we observe that

$$
\begin{aligned}
& \frac{\operatorname{Li}_{k}\left(1-e^{-t}\right)}{\log (1+t)}(1+t)^{x} \\
& \quad=\sum_{n=0}^{\infty} b_{n}^{(k)}(x) \frac{t^{n}}{n !} \\
& =\frac{1}{\log (1+t)} \underbrace{\int_{0}^{t} \frac{1}{e^{x}-1} \int_{0}^{t} \frac{1}{e^{x}-1} \cdots \frac{1}{e^{x}-1}}_{k-1 \text { times }} \int_{0}^{t} \frac{x}{e^{x}-1} d x \cdots d x(1+t)^{x} .
\end{aligned}
$$

Thus, by (11), we get

$$
\begin{aligned}
\sum_{n=0}^{\infty} b_{n}^{(2)}(x) \frac{t^{n}}{n !} & =\frac{(1+t)^{x}}{\log (1+t)} \int_{0}^{t} \frac{x}{e^{x}-1} d x \\
& =\frac{(1+t)^{x}}{\log (1+t)} \sum_{l=0}^{\infty} \frac{B_{l}}{l !} \int_{0}^{t} x^{l} d x \\
& =\left(\frac{t}{\log (1+t)}\right)(1+t)^{x} \sum_{l=0}^{\infty} \frac{B_{l}}{(l+1)} \frac{t^{l}}{l !} \\
& =\sum_{n=0}^{\infty}\left\{\sum_{l=0}^{n}\left(\begin{array}{l}
n \\
l
\end{array}\right) \frac{B_{l} b_{n-l}(x)}{l+1}\right\} \frac{t^{n}}{n !} .
\end{aligned}
$$

Therefore, by (12), we obtain the following theorem.

Theorem 2.1 For $n \geq 0$ we have

$$
b_{n}^{(2)}(x)=\sum_{l=0}^{n}\left(\begin{array}{l}
n \\
l
\end{array}\right) \frac{B_{l} b_{n-l}(x)}{l+1} .
$$

From (11), we have

$$
\begin{aligned}
\sum_{n=0}^{\infty} b_{n}^{(k)}(x) \frac{t^{n}}{n !} & =\frac{\operatorname{Li}_{k}\left(1-e^{-t}\right)}{\log (1+t)}(1+t)^{x} \\
& =\frac{t}{\log (1+t)} \frac{\operatorname{Li}_{k}\left(1-e^{-t}\right)}{t}(1+t)^{x}
\end{aligned}
$$

We observe that

$$
\begin{aligned}
\frac{1}{t} \operatorname{Li}_{k}\left(1-e^{-t}\right) & =\frac{1}{t} \sum_{n=1}^{\infty} \frac{1}{n^{k}}\left(1-e^{-t}\right)^{n} \\
& =\frac{1}{t} \sum_{n=1}^{\infty} \frac{(-1)^{n}}{n^{k}} n ! \sum_{l=n}^{\infty} S_{2}(l, n) \frac{(-t)^{l}}{l !} \\
& =\frac{1}{t} \sum_{l=1}^{\infty} \sum_{n=1}^{l} \frac{(-1)^{n+l}}{n^{k}} n ! S_{2}(l, n) \frac{t^{l}}{l !} \\
& =\sum_{l=0}^{\infty} \sum_{n=1}^{l+1} \frac{(-1)^{n+l+1}}{n^{k}} n ! \frac{S_{2}(l+1, n)}{l+1} \frac{t^{l}}{l !} .
\end{aligned}
$$


Thus, by (10) and (14), we get

$$
\begin{aligned}
\sum_{n=0}^{\infty} b_{n}^{(k)}(x) \frac{t^{n}}{n !} & =\left(\sum_{m=0}^{\infty} b_{m}(x) \frac{t^{m}}{m !}\right)\left\{\sum_{l=0}^{\infty}\left(\sum_{p=1}^{l+1} \frac{(-1)^{p+l+1}}{p^{k}} p ! \frac{S_{2}(l+1, p)}{l+1}\right) \frac{t^{l}}{l !}\right\} \\
& =\sum_{n=0}^{\infty}\left\{\sum_{l=0}^{n}\left(\begin{array}{l}
n \\
l
\end{array}\right)\left(\sum_{p=1}^{l+1} \frac{(-1)^{p+l+1} p !}{p^{k}} \frac{S_{2}(l+1, p)}{l+1}\right) b_{n-l}(x)\right\} \frac{t^{n}}{n !}
\end{aligned}
$$

Therefore, by (15), we obtain the following theorem.

Theorem 2.2 For $n \geq 0$, we have

$$
b_{n}^{(k)}(x)=\sum_{l=0}^{n}\left(\begin{array}{l}
n \\
l
\end{array}\right)\left(\sum_{p=1}^{l+1} \frac{(-1)^{p+l+1}}{p^{k}} p ! \frac{S_{2}(l+1, p)}{l+1}\right) b_{n-l}(x) .
$$

By (7), we get

$$
\begin{aligned}
& \sum_{n=0}^{\infty}\left(b_{n}^{(k)}(x+1)-b_{n}^{(k)}(x)\right) \frac{t^{n}}{n !}=\frac{\operatorname{Li}_{k}\left(1-e^{-t}\right)}{\log (1+t)}(1+t)^{x+1}-\frac{\operatorname{Li}_{k}\left(1-e^{-t}\right)}{\log (1+t)}(1+t)^{x} \\
& =\frac{t \operatorname{Li}_{k}\left(1-e^{-t}\right)}{\log (1+t)}(1+t)^{x} \\
& =\left(\frac{t}{\log (1+t)}(1+t)^{x}\right) \operatorname{Li}_{k}\left(1-e^{-t}\right) \\
& =\left(\sum_{l=0}^{\infty} \frac{b_{l}(x)}{l !} t^{l}\right)\left\{\sum_{p=1}^{\infty}\left(\sum_{m=1}^{p} \frac{(-1)^{m+p} m !}{m^{k}} S_{2}(p, m)\right)\right\} \frac{t^{p}}{p !} \\
& =\sum_{n=1}^{\infty}\left(\sum_{p=1}^{n} \sum_{m=1}^{p} \frac{(-1)^{m+p}}{m^{k}} m ! S_{2}(p, m) \frac{b_{n-p}(x) n !}{(n-p) ! p !}\right) \frac{t^{n}}{n !} \\
& =\sum_{n=1}^{\infty}\left\{\sum_{p=1}^{n} \sum_{m=1}^{p}\left(\begin{array}{l}
n \\
p
\end{array}\right) \frac{(-1)^{m+p} m !}{m^{k}} S_{2}(p, m) b_{n-p}(x)\right\} \frac{t^{n}}{n !} .
\end{aligned}
$$

Therefore, by (16), we obtain the following theorem.

Theorem 2.3 For $n \geq 1$, we have

$$
b_{n}^{(k)}(x+1)-b_{n}^{(k)}(x)=\sum_{p=1}^{n} \sum_{m=1}^{p}\left(\begin{array}{l}
n \\
p
\end{array}\right) \frac{(-1)^{m+p} m !}{m^{k}} S_{2}(p, m) b_{n-p}(x) .
$$

From (13), we have

$$
\begin{aligned}
\sum_{n=0}^{\infty} b_{n}^{(k)}(x+y) \frac{t^{n}}{n !} & =\left(\frac{\operatorname{Li}_{k}\left(1-e^{-t}\right)}{\log (1+t)}\right)^{k}(1+t)^{x+y} \\
& =\left(\frac{\operatorname{Li}_{k}\left(1-e^{-t}\right)}{\log (1+t)}\right)^{k}(1+t)^{x}(1+t)^{y} \\
& =\left(\sum_{l=0}^{\infty} b_{l}^{(k)}(x) \frac{t^{l}}{l !}\right)\left(\sum_{m=0}^{\infty}(y)_{m} \frac{t^{m}}{m !}\right)
\end{aligned}
$$




$$
\begin{aligned}
& =\sum_{n=0}^{\infty}\left(\sum_{l=0}^{n}(y)_{l} b_{n-l}^{(k)}(x) \frac{n !}{(n-l) ! l !}\right) \frac{t^{n}}{n !} \\
& =\sum_{n=0}^{\infty}\left(\sum_{l=0}^{n}\left(\begin{array}{l}
n \\
l
\end{array}\right) b_{n-l}^{(k)}(x)(y)_{l}\right) \frac{t^{n}}{n !} .
\end{aligned}
$$

Therefore, by (17), we obtain the following theorem.

Theorem 2.4 For $n \geq 0$, we have

$$
b_{n}^{(k)}(x+y)=\sum_{l=0}^{n}\left(\begin{array}{l}
n \\
l
\end{array}\right) b_{n-l}^{(k)}(x)(y)_{l} .
$$

\section{Competing interests}

The authors declare that they have no competing interests.

\section{Authors' contributions}

All authors contributed equally to this work. All authors read and approved the final manuscript.

\section{Author details}

'Department of Mathematics, Kwangwoon University, Seoul, 139-701, Republic of Korea. ${ }^{2}$ Division of General Education, Kwangwoon University, Seoul, 139-701, Republic of Korea. ${ }^{3}$ Department of Applied Mathematics, Pukyong National University, Pusan, 698-737, Republic of Korea.

\section{Acknowledgements}

The present research has been conducted by the Research Grant of Kwangwoon University in 2014.

Received: 23 June 2014 Accepted: 24 July 2014 Published: 05 Aug 2014

\section{References}

1. Kim, DS, Kim, T, Lee, S-H: Poly-Cauchy numbers and polynomials with umbral calculus viewpoint. Int. J. Math. Anal. 7, 2235-2253 (2013)

2. Prabhakar, TR, Gupta, S: Bernoulli polynomials of the second kind and general order. Indian J. Pure Appl. Math. 11 1361-1368 (1980)

3. Roman, S, Rota, GC: The umbral calculus. Adv. Math. 27(2), 95-188 (1978)

4. Choi, J, Kim, DS, Kim, T, Kim, YH: Some arithmetic identities on Bernoulli and Euler numbers arising from the $p$-adic integrals on $Z_{p}$. Adv. Stud. Contemp. Math. 22, 239-247 (2012)

5. Ding, D, Yang, J: Some identities related to the Apostol-Euler and Apostol-Bernoulli polynomials. Adv. Stud. Contemp. Math. 20, 7-21 (2010)

6. Gaboury, S, Tremblay, R, Fugère, B-J: Some explicit formulas for certain new classes of Bernoulli, Euler and Genocchi polynomials. Proc. Jangjeon Math. Soc. 17, 115-123 (2014)

7. King, D, Lee, SJ, Park, L-W, Rim, S-H: On the twisted weak weight q-Bernoulli polynomials and numbers. Proc. Jangjeon Math. Soc. 16, 195-201 (2013)

8. Kim, DS, Kim, T, Lee, S-H: A note on poly-Bernoulli polynomials arising from umbral calculus. Adv. Stud. Theor. Phys. 7(15), 731-744 (2013)

9. Kim, DS, Kim, T: Higher-order Frobenius-Euler and poly-Bernoulli mixed-type polynomials. Adv. Differ. Equ. 2013,251 (2013)

10. Kim, DS, Kim, T, Lee, S-H, Rim, S-H: Umbral calculus and Euler polynomials. Ars Comb. 112, 293-306 (2013)

11. Kim, DS, Kim, T: Higher-order Cauchy of first kind and poly-Cauchy of the first kind mixed type polynomials. Adv. Stud. Contemp. Math. 23(4), 621-636 (2013)

12. Kim, T: $q$-Bernoulli numbers and polynomials associated with Gaussian binomial coefficients. Russ. J. Math. Phys. 15 51-57 (2008)

13. Kim, Y-H, Hwang, K-W: Symmetry of power sum and twisted Bernoulli polynomials. Adv. Stud. Contemp. Math. 18 127-133 (2009)

14. Ozden, H, Cangul, IN, Simsek, Y: Remarks on q-Bernoulli numbers associated with Daehee numbers. Adv. Stud. Contemp. Math. 18, 41-48 (2009)

15. Park, J-W: New approach to q-Bernoulli polynomials with weight or weak weight. Adv. Stud. Contemp. Math. 24(1), 39-44 (2014)

16. Roman, S: The Umbral Calculus. Pure and Applied Mathematics, vol. 111. Academic Press, New York (1984). ISBN:0-12-594380-6

17. Simsek, Y: Generating functions of the twisted Bernoulli numbers and polynomials associated with their interpolation functions. Adv. Stud. Contemp. Math. 16, 251-278 (2008)

18. Srivastava, HM, Kim, T, Simsek, Y: $q$-Bernoulli numbers and polynomials associated with multiple $q$-zeta functions and basic L-series. Russ. J. Math. Phys. 12, 241-278 (2005) 
10.1186/1687-1847-2014-219

Cite this article as: Kim et al.: A note on poly-Bernoulli numbers and polynomials of the second kind. Advances in Difference Equations 2014, 2014:219

Submit your manuscript to a SpringerOpen ${ }^{\circ}$ journal and benefit from:

- Convenient online submission

- Rigorous peer review

- Immediate publication on acceptance

- Open access: articles freely available online

- High visibility within the field

- Retaining the copyright to your article

Submit your next manuscript at $\gg$ springeropen.com 